adaptation will be discussed. While sex, condition of refraction and pigmentation are factors, their influence is not of sufficient importance to require that they be taken into account in diagnosis.

\title{
Summary
}

In the foregoing paper the light sense and the amount and rapidity of dark adaptation are studied in relation to the needs and requirements in clinical work. Values are given for the light minimum on 206 eyes at ages ranging from 9 to 70 years, 114 male and 92 female. Determinations were made at the end of a standardized period of light adaptation and after 1, 2, 5, 10, 15 and 20 minutes of dark adaptation. Curves were plotted to show the distribution and range of scatter of the results obtained and a brief statistical treatment is given to bring out points of interest and value in diagnosis.

For the purpose of determining norms for clinical use the observers were divided into two groups-those under and those over 35 years of age. The 5 percentile values were selected as critical or borderline, and were plotted in the form of a curve to be used as a standard in the examination of new cases. Two record sheets are shown, one for each of the age groups, on which this curve was plotted and in addition two other curves, representing respectively the median and the 95 percentile values, in order better to aid in the orientation of the results of any new case examined in relation to those obtained from a group of non-pathological cases. These record sheets should be supplied with the instrument used for making the test.

\section{PRIMARY GLAUCOMA \\ The Respective Values of the Different Forms of Treatment of this Disease}

BY

G. Herbert Burnham, M.D.(Tor.), F.R.C.S.(Edin.) TORONTO, CANADA

This disease, primary glaucoma, as we know, is the subject of much debate as to its cause and as to the most effective manner of treatment.

With your permission I shall divide this disease into two kinds, viz., that which is associated with non-cupping of the optic disc, and that form which has cupping of the optic disc. 
The non-cupping variety passes through many stages, terminating with the acute inflammatory type, as to one division and a painless gradual loss of vision as to the other division of this type. The former progresses with varying degrees of severity of transitory attacks of plus tension and pain, till finally it passes into acute inflammatory glaucoma.

The other division of this type is associated with a gradual deterioration of sight and sometimes a little pain, rather an uneasiness, plus tension and very seldom an acute attack with pain. The length of time varies greatly in this gradual lessening of vision, namely one year or more.

In these two divisions of this type there is every degree of plus tension from the least to the greatest. The optic disc, however, does not show any signs of cupping, that is, the optic disc is the same as regards cupping, in the beginning of this disease as later on, and also even when there is $\mathrm{T} .+2$ or $\mathrm{T} .+3$. Also in the long-standing high plus tension of acute sympathetic ophthalmia, there is no cupping of the optic disc, as I have been able to verify in two typical cases of this disease, which under "The Combined Treatment" have recovered with good vision and with a clear view of the optic disc. Hence, the following conclusion might rightly be drawn, that plus tension cannot be held responsible for the cupping of the optic disc, unless one ignores much palpable evidence to the contrary. The preliminary stages of both divisions are usually treated with eserine drops chiefly and rest and later an operation, as iridectomy or one kindred in effect. When the disease, in spite of the use of the nonoperative treatment becomes more severe, then the point is reached when the condition of plus tension and pain is relieved by one of the many operations advised. Sometimes the outcome is brilliant, if done at a certain stage of the disease, but when otherwise, the result is unsatisfactory. Then the only remedy left is another operation. Now the eye is in a hazardous condition and the list of the oculist's remedies has come to an end. Now the course is palliative and rest, in other words, the limit of the oculist's list is reached and he becomes an observer and onlooker merely, according to the present line of thought and action. A patient often then asks: "Will the other eye, which is now apparently normal, become similarly affected?" Truth compels the oculist to say "Yes," but we expect with a happier termination, unless the glaucoma is of the second division, when he will have to qualify his prognosis, as the treatment of this division is somewhat precarious.

This confession, seems to me, to be a proof that there is a decided lack of thoroughness in the treatment practised. My opinion is that the treatment of both divisions is defective, and 
so convinced am I of the truth of this belief, that I have given up this mode of treatment. My only exception is an iridectomy in the very last stage of acute glaucoma and even then I do use, in addition, internal medicinal, remedies to ensure success.

In regard to the variety with cupping of the optic disc the progress of the disease is gradual, painless, and ends practically in blindness, in spite of all the usual resources of treatment recognized and practised by oculists. The tension in these cases is plus and of such a character, that it seems unreasonable that it can be the cause of such deep cupping, especially of that seen in advanced stages. The usual treatment fails as a rule to stop the progress of the disease. The line of treatment consists of drops of various kinds and operations to relieve plus tension and to restore normal tension; for so unanimous are the oculists in ascribing the cupping and progress of the disease to plus tension.

The results of these operations seem to be a strong support of the belief that the treatment, to which oculists cling, is not correct in principle. It is rightly stated that the operative treatment of glaucoma is in a certain type of non-cupping though brilliant, uncertain after all.

In other non-cupping types the operative treatment may be successful, but the oculist cannot be sure; for he knows that he has often been sadly disappointed. Then further to emphasize my belief, that the whole line of treatment is apparently wanting in scope, is that the patient has to be told that the other eye will become affected sooner or later, and besides, that there is no actual preventive that can be said to be able to save the eye from the same disease that attacked the eye first affected.

This opinion may seem to give the impression of arraigning as ineffective the system of treatment, which has received the support of oculists for many years. If this thought be entertained, allow me to state that this disagreement with the views and practices of other oculists is founded, not only upon the results of this, another form of treatment quite different from theirs, and upon the success which has followed its use over a period of many years; but also upon my conception of the disease itself, which view has always strongly appealed to me, and has influenced and guided me in regard to treatment, till I have finally reached the decision arrived at in this paper. This statement of mine though previously made, but not with the same fullness of detail, may be thought to be rather sanguine and speculative; but the reasonableness of this conclusion of mine I shall now endeavour to establish by quoting facts and cases and by the inferences drawn from them.

The non-cupping variety of glaucoma is due, I think, to a certain type of cyclitis without the exudation of ordinary cyclitis, 
but with the property of causing plus tension, which in the first division gives rise to increasing tension, till it ends in $\mathrm{T} .+2$, or greater. In the second division it is limited to a moderate tension, variable and sometimes absent. In the beginning of the first division and almost up to the onset of the acute attack and severer pain, I have found that the disease could be got under control, in fact, could be cured, by eserine drops gr. 1/16 to $1 / 8$ ad one ounce, 2.d. to the eye and pulv. hyd. $\bar{c}$. cret. : et Doveri, one 2.d.s. one half-hour after eating and sodae iodidi, gr. 10, and sodae bromidi gr. 5, 3.d.s. one hour after eating. If at a more advanced stage I advise at once the use of "The Combined Treatment," or if at any time a satisfactory effect is not shown by the above medicinal treatment. In bad acute glaucomatous cases with much tension and pain, I do an iridectomy, and by giving, in addition, mercury and the iodide I make certain of a good recovery; for often after relief from pain and improvement of vision by operation, there begins a deterioration, which is halted by the added internal medicinal treatment. So thus you see, that one eliminates operative measures, save in the acute form with $\mathrm{T} .+1$ or $\mathrm{T} .+2$ and severe pain.

The variety of glaucoma, associated with cupping of the optic disc, I think, is not due to a cyclitis to begin with, but rather its origin is owing to an inflammation of the optic disc and of the nerve immediately behind it. Later on it gradually involves the ciliary region more in some cases than it does in others. An operation will, but not always by any means, be partially successful. But from my point of view I disapprove of any form of operation, for the plus tension as a reason is negligible. In cases. where though the cupping is quite marked but the lustre of the nerve head is good and the appearance fuzzy, the result of the following treatment is most encouraging, namely : eserine drops, mercury and the iodide, and bromide of soda; for you can see the cup gradually becoming more healthy-looking and shallower, and the field steadily improving. But when the cup is deep and its bottom has a bright glistening look, I begin with "The Combined Treatment ;" for it is necessary at once to use the most powerful remedies. When, however, the tension is plus and the eye has to the finger-tips a doughy feeling, even though the vision is 6/9 almost, the prognosis is bad; for it means such extensive disease of the whole eye, that the sclera is involved. The fellow eye, however, with $6 / 12$ vision and $T$. plus but a firm sensation with a certain elasticity of sclera will gradually deteriorate, but so slowly compared with the other eye, that the eye with vision $6 / 9$ will and did become blind, whereas the other with 6/12 vision, recently seen by me after the lapse of four years, enables the patient to distinguish faces and readily go 
about the house. The left eye four years ago held exactly the same vision as today, and the other, the right eye, was also blind then as it is today. The cup showed a bright glistening appearance about the same in each eye, which indicated a condition so serious, that ultimately there would be blindness or at the best poor vision in spite of any treatment, for the tissues of the optic disc and nerve are so hopelessly diseased.

My conclusions are, that in the beginning nearly all cases of the non-cupping and cupping varieties of glaucoma can, with few exceptions, be cured by the use of eserine, mercury and the iodide and bromide of soda. If, however, the improvement is not satisfactory then use "The Combined Treatment," for the latter treatment is much more powerful. This mode of treatment I consider more to be relied upon in every way than the treatment by operation. In regard to the cupping variety I am satisfied, that in the beginning, the medicinal treatment or " The Combined Treatment" is superior to the operative. When, however, this variety shows from the appearance of the cup that it is of long duration, even though the vision is fairly good, the result of treatment can only delay the final stage of almost blindness and in some cases blindness itself. However, sometimes I have secured a comparatively good result where I had despaired. Therefore this treatment is always justifiably used in these cases where there is any room for doubt, before finally deciding that the case is beyond help.

In the non-cupping variety, if one eye only is diseased and the other apparently not, this treatment will prevent the onset of glaucoma in the good eye. This result shows how wide and beneficial is the action of the non-operative system, as advocated by me. In the cupping variety I regard the medicinal treatment as the most trustworthy. My feeling is that the operative treatment is just about useless, being used as a last and the only resort with little belief in its ability to influence the disease.

Now with my present idea of treatment I no longer dread any ordinary case of glaucoma; for I have had such good recoveries with a continuous improvement from the beginning of the treatment.

One case of a non-cupping variety that I should like to mention is that of a man, J. R., of Toronto, aged 70 years. On examination I found right eye T.+2-, hare nercention of light in outer fielt, cornea steamy, pupil dilated, etc. Left eye was $T$. plus, vision $6 / 9$ poorly, and the usual glaucomatous field. He told me that when the sight of the left eye began to fail and was acting in the same way as the right eye did, that he was alarmed. He was fond of pigeon-shooting and became uneasy when he found that he could not see the bird in all parts of his field, as he swept his gun around". "The Combined Treatment" saved the right eye from excision, but not from blindness, and as to the left eye it became normal. In this case at the end of four years the eyes were in the same condition as when the treatment was stopped, his general health was also greatly improved as is usually the case with "The Combined Treatment." 
My treatment of this case, had I used the usual routine, would have been to have excised the right eye and to have done an operation upon the left eye, one of the many so warmly advocated. Any one of these would have at the time restored the eye to T.n., and might have given vision of $6 / 9$, but no more for after an operation, the vision heretofore present, as in this case, would likely become lessenea. Then, as to the eye's future, whether he could count on a fully restored eye or had to fear a slow relapse would have had a doubtful answer given, and besides, his general health would have remained the same, or perhaps, have become worse.

The picture of the result of "The Combined Treatment" is right eye retained, but blind, left eye $6 / 6$ vision and a good prognosis without any reservation and excellent health and spirits.

I shall now give some of my notes regarding a case of the cupping of the optic disc variety.

Miss W., aged 48 years, Toronto, first. consulted me November 15,1915 . It was a case of refraction, and the glasses she was wearing were decidedly incorrect. There was hyperopic astigmatism and presbyopia. I gave her glasses, first using homatropine as the eyes showed much retinal irritation. Each eye T.n., ps. a., optic discs normal, fields normal, and vision with glasses $6 / 6$ not fully each eye.

December 24, 1923. Since the above date, 1915, this patient has not been seen by me. She says the glasses were quite comfortable. Now she complains of a dull, heavy feeling of the left eye. She says that on and off for four years the left eye was troublesome and, when she was very tired, saw rainbow colours. At times she says that the eye was quite easy. Right eye 6/6 not fully with glasses, T.n., p.a. optic disc normal, and field normal ; left eye T.n., vision 6/15 with glasses, pupil slowly active and does not contract as fully as right eye, and the pupillary margin has a distinctly atrophic look, media clear, optic disc deep cup slightly shelving towards outer edge, and nasal field lost, general health poor. The treatment prescribed was eserine drops gr. $1 / 16$ to $1 / 8$, ad one ounce $2 . d$. into left eye, pulv. hyd. $\bar{c}$ cret., et Doveri, 2d.s. half an hour after eating, and sodae iodidi gr. 10 and sodae bromidi gr. 5, 3.d.s. one hour after eating. She continued the treatment till June, 1924, when she contracted pneumonia and died after three days' illness. The patient visited me one week before her death and was quite well. Left eye was as follows: T.n., p.a., but does not contract quite as well as right pupil, the atrophic look of the margin of the pupil has gone, and the normal nasal field almost restored, media clear, vision 6/6 not fully with glasses. There is still a marked cupping of the optic disc, but it is healthier looking as to lustre and has a fuzzy appearance instead of a glistening effect, is shallower, general health is excellent.

As the improvement was so steady and so satisfactory I had not used "The Combined Treatment," but if at any time had there been a slowing down as regards improvement I should at once have done so. The improvement of the eye and general health was so well sustained, that the patient, now aged 57 years, was very pleased and frequently remarked upon it. Had I been able further to proceed with my treatment I should have watched with much interest the recovery of the optic disc; for it was gradually and noticeably getting healthier-looking and shallower.

It seems to me that I have a right to attribute the improvement in the aspect of the optic disc to the treatment, and I think that finally its condition would have resembled closely a physiological cup. And why should it not have gone on improving with the continuance of the treatment, till it became as before the attack, if not exactly a normal optic disc, still one that would have had the resemblance and power of a normal physiological cup. It does appear to me that the tension manifestly could not have been the cause of the cupping. The appearance of the pupil and iris showed that the pathological changes, apparently begun in the optic nerve, had slowly in four years or more, crept forwards, till the ciliary region was implicated. The usual treatment would 
of course have been an operation on the left eye with a doubtful prognosis as to its future. Surely the operative treatment ought to have enabled the oculist with assurance to have given a good prognosis, if an operation in these cases was ever going to be able to be considered, as radically effective.

The operative treatment besides giving a doubtful prognosis as regards the future of the eye could only express with regard to the right eye the hope that it would not be affected. Whereas the treatment outlined in this paper and its results would warrant a well-founded, decidedly favourable prognosis as to the left eye, and as to the right eye freedom from any glaucomatous attack.

In regard to the loss of the nasal field in both types of glaucoma this condition is thought to be associated with plus terision, in fact, is so asserted by oculists. In the non-cupping variety the plus tension appears to be due to the cyclitis, which interferes with the circulation of the angle of the anterior chamber. Hence its slight and evanescent character, as a rule, to begin with and the fact that, with no treatment or with unsuitable treatment and faulty hygienic control of the eyes, it becomes greater and more persistent as the disease progresses. Then, that an operation at a certain stage seems to cure, at another relieves and does not cure, is due to the condition of the ciliary region as to the duration and severity of the disease of that region. In these cases the medicinal treatment brings about a recovery by its action upon the diseased ciliary region. In the cupping variety of glaucoma there is a plus tension, very moderate in character and sometimes absent. One is surely pardoned if he fails to grasp, to understand the line of thought of those who declare that the cupping of the optic disc is due to the pressure of plus tension, when in another form of glaucoma in which the optic disc is subjected to the pressure of a plus tension slight to begin with, but finally after a time is increased to $T .+2$ and even $T .+3$; there is not produced at any stage of the plus tension any sign of cupping of the optic disc.

In the cupping variety of glaucoma the loss of the nasal field seems to me to be due to local pathological changes in the optic nerve. In this form the plus tension is moderate and is apparently due to involvement of the ciliary region which cyclitis does not bring about the same radical change in the angle of the anterior chamber, as it does in the non-cupping variety.

In cases of retro-ocular neuritis there is no cupping of the optic disc and no increase of tension, with a central scotoma and besides, the optic disc has a normal appearance long after the beginning of the neuritis. As the pathological changes taking place in the optic nerve in one of these diseases cause cupping of the optic disc and loss of the nasal field, and in the other no semblance of 
cupping and a scotoma $a_{1}$ it seems as if the difference can only be accounted for, but not as yet explained, in the peculiar character of the neuritis. Here I am strongly of the opinion is a most interesting field for pathological research and one far reaching as regards the deductions which will naturally follow its successful working out.

In the non-cupping variety of glaucoma the nasal field is lessened or lost through a plus tension, its presence arising as it does from changes in the ciliary region, that is, the anterior part of the eye. Thus this condition is a question presumably chiefly of mechanical pressure, which can consequently be so sudden and so destructive in its effects and is not associated with any marked pathological change in the optic disc. The loss of the nasal field in the cupping variety is due to local changes in the optic nerve and not to pressure, whereas in the non-cupping the nasal field is lost through pressure.

Thus it is evident that in the non-cupping an operation can be of benefit and that in the cupping it is of such questionable value. Of the cupping variety of glaucoma and of retro-ocular neuritis the medicinal treatment in which term is always included "The Combined Treatment" does bring about a cure if used in the early stages of these diseases; but if later, then though the healing of the diseased tissues takes place often it is too late to restore these tissues to normalcy.

However, "The Combined Treatment" does prevent the extension of these diseases to kindred tissues of the other eye by healing the diseased tissue of this, the affected eye. Thus, it prevents the infection of the so-called healthy eye, and also it has a salutary action upon the good eye itself. This idea, rather this fact, thus upholds my theory of transmission of an injurious influence along the kindred nerves to the other eye, as in the case of acute sympathetic ophthalmia, and also of a healing influence along these same nerves. This healing influence is really forced into greatly heightened and sustained activity through the use of "The Combined Treatment" (for remember, it never causes an unhealthy influence), and thus it is enabled ultimately to overpower the one-time stronger, inimical influence. Moreover, we know that this abnormal influence can be quickly checked if attacked early in the disease, but otherwise the contest waged is very great and long before this malign influence is overcome and victory is at last won; for this pernicious influence, as proved, has been and can again be subdued, and thereby rendered inert and innocuous.

Therefore, it seems to me to be a natural sequence to express the opinion, that the comparison regarding the merits of the respective treatments of glaucoma is not overdrawn; but is a wellbalanced résumé, which appears to me to justify the conclusion 
that the internal medicinal treatment, as advocated in this article, is superior in many ways to and more to be relied upon than the usual modes of treatment of this disease-primary glaucoma.

\title{
REFERENCES
}

1. Sympathetic Ophthalmia. The Successful Treatment of a Patient with an Acute Case. G. Herbert Burnham, M.D.(Tor.), F.R.C.S.(Edin.). Arch. of Ophthal,., February, 1930.

2. "The Combined Treatment" of Certain Diseases of the Eye. Arch. of Ophthal., February, 1934.

(In this paper there is given by request of oculists a detailed account of the mode of administration of this treatment).

\section{THREE UNUSUAL CASES OF FOREIGN BODIES IN AND ABOUT T.HE EYEBALL*}

BY

\section{FREDERICK T. TOOKE}

\author{
MONTREAL, CANADA
}

To those who attended the meeting of this Society held at Hot Springs in 1920, or to others who may have read its transactions for that year, the identically similar title of this, with my previous paper, will immediately be apparent. The earlier article was presented with the more particular appeal, and possibly with some unusual element of interest, to those who had seen service in the Army Medical Corps. The value of the paper, however, as in so many presented before this Society, consisted, not so much in the article itself, as in the interesting and varied discussion and contributory elements of information which it evoked.

The cases about to be recorded occurred in civil life and are comparable to the former ones in one respect in particular, and that is, that the term "unusual" is even more emphatically applicable. The offerings are definitely alike in that the foreign bodies were retained for a considerable period of time, but absolutely incomparable regarding the nature or character of the accident and the quality of the material responsible for each injury.

I acknowledge, at the outset, that as subject of an ultra-scientific character, supplied or supported with a pathological background, or possessing a bio-chemical problem, as seems to be essential in so many of the articles read at present day meetings, it is valueless. As supplying a short series of varied practical problems which the majority of us have to face, not as laboratory workers, but as practising ophthalmologists, the cases may possibly convey some information and possess some worth.

\footnotetext{
- Read before the American Ophthalmological Society, July 10, 1934.
} 